\title{
Effect of rikkunshito, a Japanese herbal medicine, on gastrointestinal symptoms and ghrelin levels in gastric cancer patients after gastrectomy
}

\author{
Shuji Takiguchi $\cdot$ Yuichiro Hiura $\cdot$ Tsuyoshi Takahashi $\cdot$ Yukinori Kurokawa \\ Makoto Yamasaki • Kiyokazu Nakajima · Hiroshi Miyata • Masaki Mori • \\ Hiroshi Hosoda $\cdot$ Kenji Kangawa $\cdot$ Yuichiro Doki
}

Received: 5 December 2011/ Accepted: 11 May 2012/Published online: 16 August 2012

(c) The International Gastric Cancer Association and The Japanese Gastric Cancer Association 2012

\begin{abstract}
Background Gastric cancer patients who undergo gastrectomy suffer from a post-gastrectomy syndrome that includes weight loss, dumping syndrome, reflux esophagitis, alkaline gastritis, and finally malnutrition. It is important to ameliorate the post-gastrectomy symptoms to restore postoperative quality of life (QoL). The aim of this study was to investigate the effect of rikkunshito, a Japanese herbal medicine, on postoperative symptoms and ghrelin levels in gastric cancer patients after gastrectomy. Methods Twenty-five patients who had undergone gastrectomy received $2.5 \mathrm{~g}$ of rikkunshito before every meal for 4 weeks, and a drug withdrawal period was established for the next 4 weeks. Changes in gastrointestinal hormones, including ghrelin, and appetite visual analog scale scores were measured, and QoL was estimated by using the European Organization for Research and Treatment of Cancer core questionnaire QLQ-C30. The Dysfunction After Upper Gastrointestinal Surgery for Cancer (DAUGS) scoring system was used to evaluate gastrointestinal symptoms after gastrectomy.

Results Sixteen men and nine women (mean age 61.9 years) were enrolled in the study. All patients had either stage I $(n=24)$ or II $(n=1)$ disease and had
\end{abstract}

S. Takiguchi $(\bowtie) \cdot$ Y. Hiura · T. Takahashi · Y. Kurokawa · M. Yamasaki · K. Nakajima · H. Miyata - M. Mori · Y. Doki Division of Gastroenterological Surgery,

Department of Surgery, Graduate School of Medicine, Osaka University, 2-2, E2,

Yamadaoka, Suita, Osaka 565-0871, Japan

e-mail: stakiguchi@gesurg.med.osaka-u.ac.jp

H. Hosoda $\cdot$ K. Kangawa

Department of Biochemistry, National Cardiovascular Center Research Institute, Osaka, Japan undergone either distal gastrectomy $(n=17)$ or total gastrectomy $(n=8)$ by a laparoscopy-assisted approach. The mean ratio of the acyl-/total ghrelin concentration increased significantly after rikkunshito administration (Pre: $7.8 \pm 2.1$, 4 weeks: $10.5 \pm 1.7 \%, p=0.0026$ ). The total DAUGS score, as well as the scores reflecting limited activity due to decreased food consumption, reflux symptoms, dumping symptoms, and nausea and vomiting significantly improved after rikkunshito administration.

Conclusions The present study demonstrated a significant attenuation of gastrointestinal symptoms after gastrectomy by treatment with rikkunshito. Rikkunshito is potentially useful to minimize gastrointestinal symptoms after gastrectomy.

Keywords Rikkunshito · Ghrelin · Gastric cancer · Gastrectomy

\section{Introduction}

In Japan, the number of long-term survivors after radical surgery for gastric cancer has been increasing as a result of early detection and improved surgical techniques [1, 2]. Although survivors may be rendered free of disease by surgery, they may suffer from post-gastrectomy syndrome, which includes weight loss, dumping syndrome, stasis syndrome, reflux esophagitis, alkaline gastritis, and, finally, malnutrition [3, 4]. Because of the improved prognosis of patients with gastric cancer, it is important to ameliorate post-gastrectomy symptoms to restore postoperative quality of life (QoL) [5-7].

Our group has focused on ghrelin, a gut hormone known to increase appetite [8]. Ghrelin, an endogenous ligand for the growth hormone $(\mathrm{GH})$ secretagogue receptor, displays 
dose-dependent GH-releasing activity [8, 9]. Ghrelin, which is predominantly secreted by gastric endocrine cells, stimulates food intake and triggers a positive energy balance through a central mechanism involving hypothalamic neuropeptides [10-12]. In our previous study, we reported that intravenous administration of ghrelin enhanced oral feeding and was effective against weight loss after total gastrectomy [13] and esophagectomy [14] in cancer patients.

Recently, it has been reported that rikkunshito, one of the traditional Japanese medicines, increased the plasma acylated ghrelin level in healthy volunteers and in normal mice [15]. In rats, a flavonoid in rikkunshito suppressed cisplatin-induced decreases in the plasma acylated ghrelin level and increased food intake mediated by $5-\mathrm{HT} 2 \mathrm{~B} / 2 \mathrm{C}$ receptors [16]. In general, rikkunshito is used to treat various gastrointestinal tract disorders such as functional dyspepsia (FD) [17, 18], gastro-esophageal reflux [19, 20], dyspeptic symptoms of post-gastrointestinal surgery [21, 22], and chemotherapy-induced nausea [23]. In animal experiments, rikkunshito is reported to ameliorate gastric distension via a nitric oxide-mediated pathway, and it has also been shown to improve delayed gastric emptying [24]. These results indicate that rikkunshito may decrease postoperative symptoms after gastrectomy. To tackle these issues, we conducted a prospective observational study in patients with gastric cancer after they had undergone laparoscopy-assisted gastrectomy. The aim of this study was to investigate the effect of rikkunshito on postoperative symptoms and ghrelin levels in gastric cancer patients after gastrectomy.

\section{Patients and methods}

\section{Patient eligibility}

Patients with histologically confirmed gastric cancer who had undergone gastrectomy and lymph node dissection with curative intent were eligible for participation in the study. Further criteria were: enrollment 6 months to 5 years following the surgery, ability to take solid foods, Eastern Cooperative Oncology Group performance status (PS) of $\leq 1$, age between 20 and 80 years, and adequate function of major organs. Patients were excluded from the study if they were pregnant or desired to become pregnant, undergoing chemotherapy, burdened with other active malignancy, or otherwise considered to be ineligible by the investigator. The study protocol was approved by the Human Ethics Review Committee of Osaka University School of Medicine. A signed consent form was obtained from each enrolled patient before study entry in accordance with the Declaration of Helsinki. This study was registered in the University Hospital Medical Information Network (UMIN R000006959).

Following gastrectomy, prominent postoperative symptoms and complaints tend to resolve naturally after the first 6 months. Our previous randomized study comparing dysfunction after Billroth I and Roux-en-Y reconstruction after distal gastrectomy actually revealed little difference in QoL scores and Dysfunction After Upper Gastrointestinal Surgery for Cancer (DAUGS) scores between the treatment arms after the first 6 months [25]. In order to explicitly evaluate the value of rikkunshito, therefore, all patients in the present study were recruited more than 6 months after the surgery, when various symptoms due to the gastrectomy would have more or less stabilized.

\section{Rikkunshito administration}

The study protocol is summarized in Fig. 1. Rikkunshito, which was obtained from Tsumura (Tokyo, Japan), has 8 main constituents: Glycyrrhizae radix $(4.7 \%)$, Zingiberis rhizoma $(2.3 \%)$, Atractylodis lanceae rhizoma (18.6\%), Zizyphi fructus $(9.3 \%)$, Aurantii nobilis pericarpium $(9.3 \%)$, Ginseng radix (18.6\%), Pinelliae tuber (18.6\%), and Hoelen $(18.6 \%)$. More detailed descriptions of all the substances that are known to be included in rikkunshito are found in a previous report [26]. Patients who had undergone gastrectomy invariably received $2.5 \mathrm{~g}$ of rikkunshito before each meal (7.5 g/day) for 4 weeks. After this administration period, the drug was withdrawn for the next 4 weeks.

Assessment of gastrointestinal symptoms, appetite, and QoL after gastrectomy

To evaluate gastrointestinal symptoms after the surgical resection of gastric cancer, we used the DAUGS scoring system [27]. Patients rated items related to postoperative dysfunction using a scale ranging from 1 ('not at all') to 5

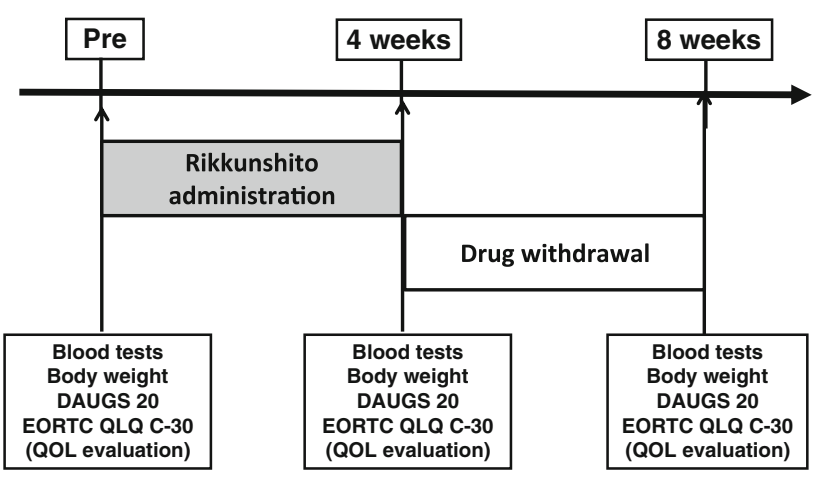

Fig. 1 Study protocol. DAUGS Dysfunction After Upper Gastrointestinal Surgery for Cancer, EORTC European Organization for Research and Treatment of Cancer, $Q O L$ quality of life 
('very severe'). The items were divided into 7 categories: (1) limited activity due to decreased food consumption, (2) reflux, (3) gastric dumping, (4) nausea and vomiting, (5) digestive difficulties, (6) pain, and (7) lower gastrointestinal (GI) symptoms [27].

We administered the European Organization for Research and Treatment of Cancer core questionnaire (QLQ-C30) [28] before and after rikkunshito administration, and after the drug withdrawal. The QLQ-C30 contains five functional scales (physical, role, cognitive, emotional, and social), three symptom scales (fatigue, pain, and nausea/vomiting), a global health/QoL scale, and six single items (dyspnea, insomnia, appetite loss, constipation, diarrhea, and financial difficulties). All scale and single items scores range from 0 to 100 . A high score for a functional scale represents a higher ("better") level of functioning, whereas a high score for a symptom scale or item represents a higher ("worse") level of symptoms [28].

Patients were instructed to rate themselves by selecting the scale at three time points; once prior to rikkunshito administration, once after the administration for 4 weeks, and once 4 weeks after the drug withdrawal.

Appetite profile was measured using a $100-\mathrm{mm}$ visual analog scale (VAS), with the questions 'How hungry are you?' and 'How full do you feel?' that were anchored with scores of ' 0 -not at all' and '100-extremely'. Patients were instructed to rate their appetite by selecting the scale that was closest to their feeling before each of three meals at three time points noted by the investigator: once prior to rikkunshito administration, once after the administration for 4 weeks, and once 4 weeks after drug withdrawal. The mean VAS score was calculated for each of the three measurement days.

To minimize bias, patients were provided with the questionnaire sheets to be filled in at home and were asked to mail them back to the data center using the specific envelopes provided.

\section{Blood sampling}

Blood samples were collected before breakfast after an overnight fastbefore and after rikkunshito administration, and after the drug withdrawal. The samples were transferred immediately into chilled tubes containing disodium ethylenediamine tetra-acetic acid (EDTA) and aprotinin for plasma sampling, centrifuged at $4{ }^{\circ} \mathrm{C}$, separated for serum sampling, and stored at $-50{ }^{\circ} \mathrm{C}$. The plasma samples were mixed with a $10 \%$ volume of $1 \mathrm{M}$ hydrochloric acid $(\mathrm{HCl})$ before storing at $-50{ }^{\circ} \mathrm{C}$. Plasma acyl- and desacyl-ghrelin concentrations were measured with a sandwich-type enzyme immunoassay kit according to the protocol supplied by the manufacturer (Mitsubishi Kagaku Iatron, Tokyo, Japan) [29]. The total plasma ghrelin concentration was calculated as the acyl-ghrelin concentration plus the desacyl-ghrelin concentration. Serum GH, insulin, and leptin concentrations were measured using a GH "Daiichi" Kit (TFB, Tokyo, Japan), a chemiluminescence enzyme immunoassay (Fujirebio, Tokyo, Japan), and a Human Leptin RIA Kit (Linco Research, St Charles, MO, USA), respectively. Serum insulin-like growth factor-1 (IGF-1) levels were measured by radioimmunoassay (RIA) (SRL,Tokyo, Japan).

Statistical analysis

Continuous variables were expressed as mean \pm SD unless otherwise stated. Statistically significant differences between the periods were calculated by paired $t$-test, Fisher's exact test, or the Mann-Whitney test. Statistical significance was set at $p<0.05$. All calculations were performed using the JMP (version 9.0) software program (SAS Institute, Cary, NC, USA).

\section{Results}

Patient characteristics

This prospective observational study focused on 25 patients with gastric cancer who had undergone gastrectomy and were enrolled between December 2010 and May 2011. Table 1 lists the demographic and clinical characteristics of the patients in this study. Sixteen men and nine women were enrolled (mean age 61.9 years). The mean body mass index (BMI) was $21.0 \pm 2.7 \mathrm{~kg} / \mathrm{m}^{2}$. All patients had either stage I $(n=24)$ or II $(n=1)$ disease and had undergone either distal gastrectomy $(n=17)$ or total gastrectomy $(n=8)$. Because early-stage cancers are indicated for a laparoscopy-assisted approach at our institution, all surgeries for patients enrolled in the study were performed by the minimally invasive approach. The mean time elapsed between surgery and enrollment was $890 \pm 578$ days.

\section{Blood tests and hormonal assays}

Table 2 summarizes the results of laboratory tests, tests of nutritional status, and hormonal assays. None of the indicators of nutritional status changed significantly after rikkunshito administration. With regard to the hormonal assays, GH (a target hormone for ghrelin), IGF-1 (a mediator of GH), insulin, and leptin also did not change significantly after rikkunshito administration. There were no significant differences in the results of other laboratory tests after rikkunshito administration compared with the results 4 weeks after drug withdrawal. 
Table 1 Patients' characteristics

\begin{tabular}{ll}
\hline$n$ & 25 \\
\hline Age (years) & $61.9 \pm 10.9$ \\
Gender (male/female) & $16 / 9$ \\
BMI $\left(\mathrm{kg} / \mathrm{m}^{2}\right.$ ) & $21.0 \pm 2.7$ \\
Operative procedure (LADG/LATG) & $17 / 8$ \\
Reconstruction (B-I/R-Y) & $15 / 10$ \\
Postoperative days (days) & $890 \pm 578$ \\
p-Stage (I/II/III/IV) & $24 / 1 / 0 / 0$ \\
\hline
\end{tabular}

Values are mean $\pm \mathrm{SD}$

$L A D G$ laparoscopy-assisted distal gastrectomy, $L A T G$ laparoscopyassisted total gastrectomy, $B M I$ body mass index, $B-I / R-Y$ Bismuth-I/ Roux-en-Y

Table 2 Results of laboratory tests, tests of nutritional status, and hormonal assays

\begin{tabular}{|c|c|c|c|}
\hline & Pre & 4 Weeks & 8 Weeks \\
\hline Hemoglobin $(\mathrm{g} / \mathrm{dl})$ & $11.2 \pm 0.8$ & $11.5 \pm 1.3$ & $11.3 \pm 1.1$ \\
\hline Albumin (g/dl) & $4.0 \pm 0.4$ & $3.9 \pm 0.3$ & $3.8 \pm 0.3$ \\
\hline Lymphocytes $(/ \mu \mathrm{l})$ & $1580 \pm 400$ & $1780 \pm 580$ & $1620 \pm 430$ \\
\hline Cholinesterase (IU/l) & $274 \pm 81$ & $275 \pm 84$ & $266 \pm 74$ \\
\hline Triglyceride (mg/dl) & $102 \pm 58$ & $92 \pm 42$ & $98 \pm 31$ \\
\hline Total cholesterol $(\mathrm{mg} / \mathrm{dl})$ & $197 \pm 28$ & $198 \pm 27$ & $202 \pm 30$ \\
\hline \multicolumn{4}{|l|}{ Rapid turnover proteins } \\
\hline Pre-albumin (mg/dl) & $26.5 \pm 7.3$ & $26.8 \pm 7.8$ & $25.8 \pm 6.4$ \\
\hline $\begin{array}{l}\text { Retinol binding protein } \\
(\mathrm{mg} / \mathrm{dl})\end{array}$ & $4.3 \pm 1.2$ & $4.3 \pm 1.2$ & $4.1 \pm 1.1$ \\
\hline Transferrin (mg/dl) & $244 \pm 35$ & $243 \pm 34$ & $229 \pm 30$ \\
\hline \multicolumn{4}{|l|}{ Hormonal assays } \\
\hline $\begin{array}{l}\text { Growth hormone (ng/ } \\
\text { ml) }\end{array}$ & $1.8 \pm 1.6$ & $1.6 \pm 0.8$ & $1.7 \pm 0.9$ \\
\hline Leptin (ng/ml) & $2.8 \pm 1.8$ & $2.5 \pm 1.5$ & $2.6 \pm 1.4$ \\
\hline \multicolumn{4}{|c|}{ Insulin-like growth factor-1 (ng/ml) } \\
\hline Insulin $(\mu \mathrm{IU} / \mathrm{ml})$ & $6.4 \pm 3.2$ & $7.2 \pm 4.1$ & $6.8 \pm 4.3$ \\
\hline
\end{tabular}

Values are mean $\pm \mathrm{SD}$

Ratio of acyl-/total ghrelin concentration and plasma acyl- and desacyl-ghrelin concentration

Figure 2 shows serial changes in the ratio of the acyl-/total ghrelin concentration after rikkunshito administration. The mean ratio of the acyl-/total ghrelin concentration increased significantly after rikkunshito administration (Pre: $7.8 \pm$ 2.1, 4 weeks: $10.5 \pm 1.7 \%, p=0.0026)$. Four weeks after the drug withdrawal, the mean ratio of the acyl-/total ghrelin concentration had decreased significantly (4 weeks: $10.5 \pm$ 1.7, 8 weeks: $7.6 \pm 2.3 \%, p=0.0015$ ). Table 3 shows the serial changes in plasma ghrelin concentrations. The administration and withdrawal of rikkunshito had no significant influence on the concentrations of acyl- and desacylghrelin. Although the difference did not reach statistical

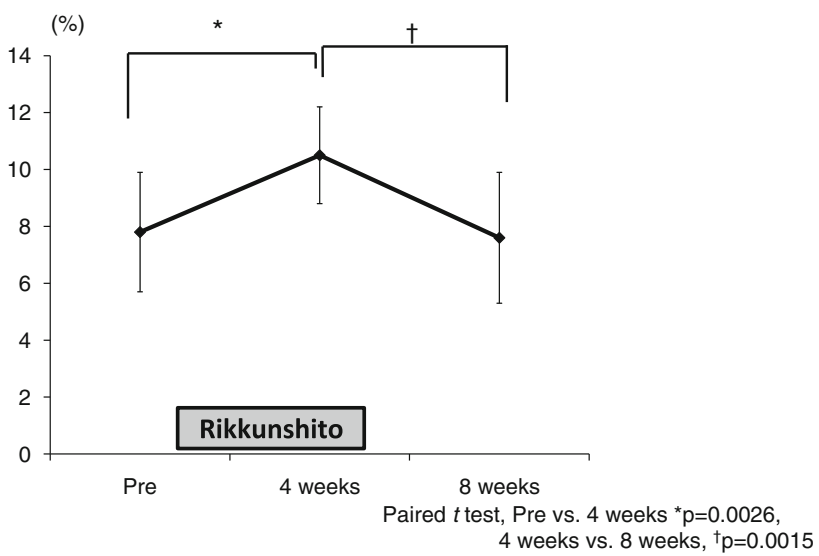

Fig. 2 Serial changes in the ratios of acyl-/total ghrelin concentration. Error bars represent SDs

Table 3 Plasma ghrelin concentrations before and after rikkunshito administration

\begin{tabular}{lccc}
\hline & Pre & 4 Weeks & 8 Weeks \\
\hline LADG & & & \\
$\begin{array}{l}\text { Acyl-ghrelin (fmol/ml) } \\
\text { Desacyl-ghrelin } \\
\text { (fmol/ml) }\end{array}$ & $5.0 \pm 2.8$ & $5.7 \pm 2.2$ & $4.9 \pm 2.4$ \\
Acyl-/total ghrelin (\%) & $7.6 \pm 3.4$ & $10.0 \pm 2.3$ & $8.2 \pm 3.4^{\dagger}$ \\
LATG & & & \\
$\begin{array}{l}\text { Acyl-ghrelin (fmol/ml) } \\
\begin{array}{l}\text { Desacyl-ghrelin } \\
\text { (fmol/ml) }\end{array}\end{array}$ & $0.89 \pm 0.49$ & $1.1 \pm 0.60$ & $0.74 \pm 0.42$ \\
Acyl-/total ghrelin (\%) & $6.8 \pm 2.2$ & $10.1 \pm 3.8$ & $12.4 \pm 3.5$ \\
\hline
\end{tabular}

Values are mean $\pm \mathrm{SD}$

* Paired $t$-test, $p<0.05$; Pre versus 4 weeks, ${ }^{\dagger} p<0.05$; 4 weeks versus 8 weeks

significance, the mean ratio of the acyl-/total ghrelin concentration was higher after rikkunshito administration in the patients who had undergone distal gastrectomy (Pre: $7.6 \pm$ 3.4, 4 weeks: $10.0 \pm 2.3 \%, p=0.075)$. In the patients who had undergone total gastrectomy, the mean ratio of the acyl-/ total ghrelin concentration was significantly increased after rikkunshito administration (Pre: $6.8 \pm 2.2$, 4 weeks: $9.8 \pm$ $2.4 \%, p=0.011)$. After the drug withdrawal, the mean ratio of the acyl-/total ghrelin concentration decreased significantly in all the patients (those who had had distal gastrectomy: 4 weeks: $10.0 \pm 2.3,8$ weeks: $8.2 \pm 3.4 \%$, $p=0.049$; those who had had total gastrectomy: 4 weeks: $9.8 \pm 2.4$, 8 weeks: $6.8 \pm 2.8 \%, p=0.009$, respectively).

Effect of rikkunshito on DAUGS score, appetite, and QoL

The VAS scores and DAUGS scores are presented in Fig. 3. The VAS scores were significantly increased after 
Fig. 3 Appetite visual analog scale (VAS) scores and DAUGS scores before and after rikkunshito administration. Higher VAS values indicate a better status for appetite, while the DAUGS scoring system evaluates better function as a lower score (see "Patients and methods"). Error bars represent SDs. GI gastrointestinal



rikkunshito administration and they decreased after the drug withdrawal (Pre: $6.6 \pm 1.9,4$ weeks: $8.6 \pm 1.2$ points, $p<0.05$; 8 weeks: $7.3 \pm 1.1$ points, $p<0.05$ compared with the score at 4 weeks). The total DAUGS score and the scores reflecting limited activity due to decreased food consumption, reflux symptoms, dumping symptoms, and nausea and vomiting were significantly improved after rikkunshito administration. Four weeks after the drug withdrawal, the total DAUGS score and the categories of limited activity due to decreased food consumption and reflux symptoms had significantly deteriorated.

The QLQ-C30 scores before rikkunshito administration, after the administration, and after the drug withdrawal are presented in Fig. 4. There were no significant differences in the global health status scores. Among the functional scales, patients scored better after rikkunshito administration and scored worse after the drug withdrawal regarding physical functioning (Pre: $86 \pm 11,4$ weeks: $96 \pm 7$, $p<0.01$; 8 weeks: $86 \pm 9, p<0.01)$. With respect to the symptom scales and items, there were no significant differences at any time points.

Association between symptomatic improvement and acyl-/total ghrelin ratio

Table 4 lists the associations between symptomatic improvement and the acyl-/total ghrelin ratio after 4 weeks' rikkunshito administration. We divided the subjects into high $(n=13)$ and low $(n=12)$ acyl-/total ghrelin groups using a cut-off value of $9.8 \%$, representing the ratio of acyl-/total ghrelin after 4 weeks' rikkunshito administration. However, there were no significant differences in the VAS appetite score, DAUGS score, or QoL score between the two groups.

\section{Discussion}

Traditional Japanese medicines (Kampo) are widely prescribed in Japan for patients with various gastrointestinal symptoms, and rikkunshito is one of the most popular Kampo medicines for these patients. Some clinical studies have reported that rikkunshito promotes recovery from anorexia in patients with FD [17, 18, 30, 31] and gastroesophageal reflux disease (GERD) symptoms [19, 20, 32]. In another study conducted in patients with advanced breast cancer, concomitant administration of rikkunshito with antiemetics such as granisetron proved to be effective against the anorexia and vomiting induced by anticancer drugs [23]. In a recent report, rikkunshito administration was shown to stimulate peripheral ghrelin secretion in rats with anorexia induced by cisplatin or selective serotonin reuptake inhibitors [16, 33]. Furthermore, oral administration of rikkunshito was shown to stimulate the secretion of ghrelin from the stomach in healthy volunteers. Also, the plasma acylated ghrelin concentration was shown to be significantly increased after rikkunshito treatment [15]. Ghrelin stimulates food intake [9] and triggers a positive energy balance through a central mechanism involving hypothalamic neuropeptides [9-12]. Taken together, these reports suggest that the induction of ghrelin could be 
Fig. 4 Quality of life scores before and after rikkunshito administration. High scores for global health status and functional scales represents a higher ("better") level of functioning, whereas a high score for a symptom scale or item represents a higher ("worse") level of symptoms (see "Patients and methods"). Error bars represent SDs
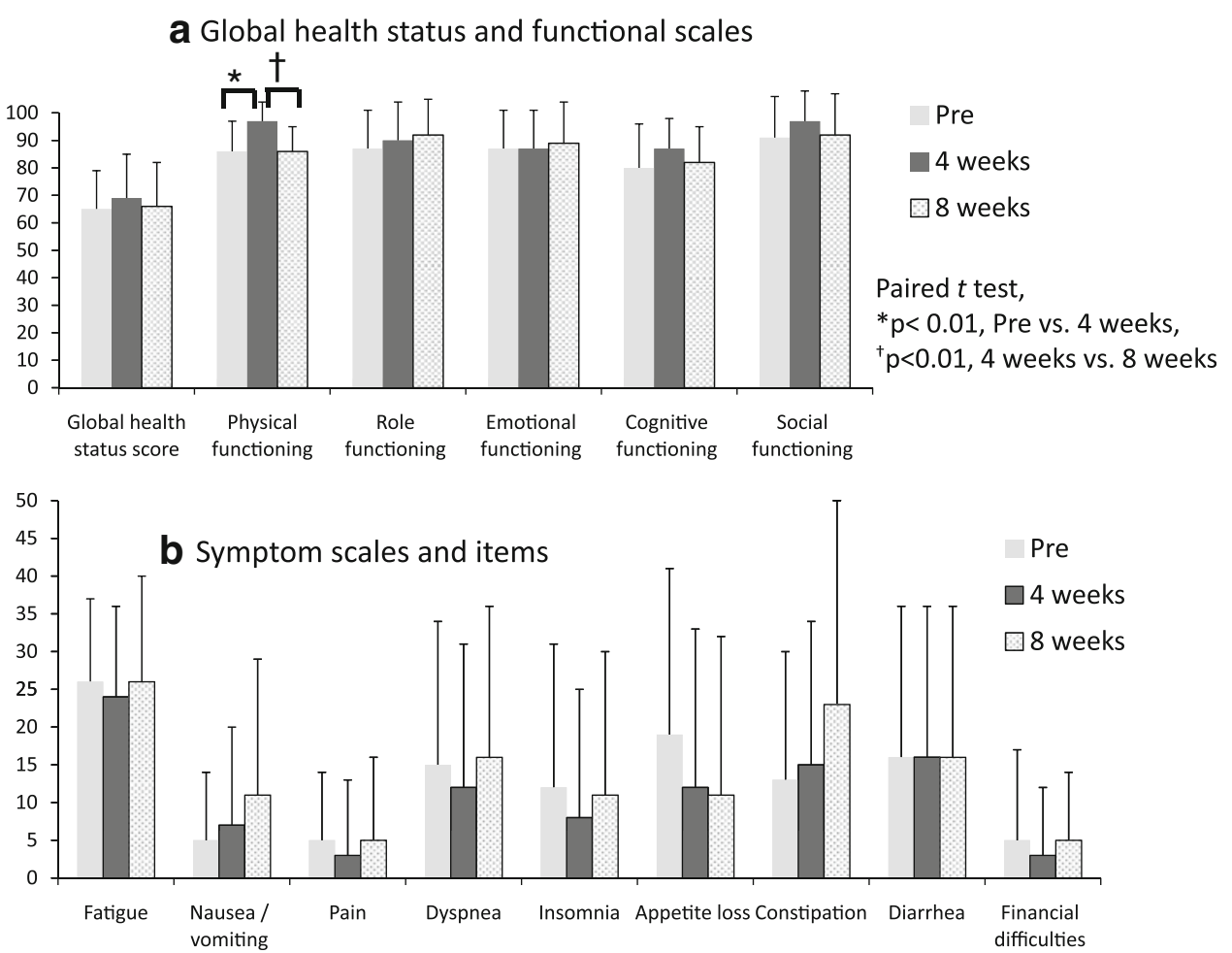

Table 4 Associations between symptomatic improvement and acylghrelin/total ghrelin ratios after rikkunshito administration

\begin{tabular}{lccc}
\hline Parameter & $\begin{array}{l}\text { High acyl-/total } \\
\text { ghrelin group }^{\mathrm{a}}\end{array}$ & $\begin{array}{l}\text { Low acyl-/total } \\
\text { ghrelin group }\end{array}$ & $p$ value \\
\hline $\begin{array}{l}\text { Appetite } \\
\text { (VAS) }\end{array}$ & $8.7 \pm 0.37$ & $8.5 \pm 0.39$ & 0.87 \\
$\begin{array}{l}\text { Total DAUGS } \\
\text { Limited } \\
\text { activity }\end{array}$ & $22.7 \pm 8.9$ & $23.5 \pm 7.4$ & 0.88 \\
$\begin{array}{l}\text { Reflux } \\
\text { symptoms }\end{array}$ & $0.47 \pm 0.62$ & $0.95 \pm 0.79$ & 0.24 \\
$\begin{array}{l}\text { Dumping } \\
\text { symptoms }\end{array}$ & $1.30 \pm 0.28$ & $1.36 \pm 0.27$ & 0.85 \\
$\begin{array}{l}\text { Nausea and } \\
\text { vomiting }\end{array}$ & $0.38 \pm 0.37$ & $0.55 \pm 0.49$ & 0.39 \\
$\begin{array}{l}\text { Functional scales }(\mathrm{QLQ}-\mathrm{C} 30) \\
\begin{array}{l}\text { Physical } \\
\text { functioning }\end{array}\end{array}$ & $92.3 \pm 7.6$ & $89.4 \pm 7.2$ & 0.33 \\
\hline
\end{tabular}

Values are mean $\pm \mathrm{SD}$

The DAUGS scoring system evaluates better function as a lower score, while higher values indicate better status for appetite (VAS) and physical functioning in the QLQ-30 (see "Patients and methods")

$D A U G S$ Dysfunction After Upper Gastrointestinal Surgery for Cancer, $V A S$ visual analog scale, $Q L Q-30$ European Organization for Research and Treatment of Cancer quality-of-life instrument for use in international clinical trials in oncology

${ }^{\text {a }}$ Acyl-/total ghrelin after rikkunshito administration $>9.8 \%$

b Acyl-/total ghrelin after rikkunshito administration $<9.8 \%$ responsible for some of the beneficial clinical effects exerted by rikkunshito.

In the present study, however, the administration of rikkunshito did not increase the ghrelin concentration per se. The difference from the previous findings with healthy volunteers could be attributed to gastrectomy, because ghrelin is predominantly secreted by gastric endocrine cells. In our previous study, a persistent decline of serum ghrelin and body weight was commonly observed after total gastrectomy [34]. In the present study, the plasma ghrelin concentration was indeed higher in the patients who had undergone distal gastrectomy than in those who had undergone total gastrectomy. It is unlikely, therefore, that rikkunshito attenuates post-gastrectomy symptoms through eliciting ghrelin secretion from the stomach.

In contrast to the serum ghrelin findings, the ratio of the acyl-/total ghrelin concentration increased significantly after treatment with rikkunshito and declined 4 weeks after the drug withdrawal. During metabolic processing, a fraction of desacyl-ghrelin is acylated in position 3 (serine) to form variants of acyl-ghrelin. Acyl-ghrelin displays dosedependent GH-releasing activity [8,9], stimulates food intake, triggers a positive energy balance [10-12], and is considered to be the active form of ghrelin [11, 35-37]. Actually, several components of rikkunshito demonstrate inhibitory activity against ghrelin desacylating enzymes [38]. In addition, 10-gingerol, a component of rikkunshito, 
inhibits exogenous ghrelin desacylation. Thus, rikkunshito may enhance the plasma acyl-ghrelin level, at least in part by inhibiting the circulating ghrelin degrading enzyme [39]. These findings, taken together, suggest that the rise in the ratio of the acyl-/total ghrelin concentration could be responsible for the beneficial effect of rikkunshito in terms of the attenuation of gastrointestinal symptoms.

Rikkunshito was reported to significantly accelerate gastric emptying and reduce gastrointestinal symptoms in adult patients with chronic idiopathic dyspepsia [17]. In another study, it was reported that, postoperatively, dyspeptic pediatric patients exhibited symptomatic relief and a significant reduction in the mean symptom score after treatment with rikkunshito over a 1-month period [21]. In the sphere of gastrointestinal surgery, rikkunshito improved gastric emptying and ameliorated the postoperative symptoms of patients who had undergone a pyloruspreserving gastrectomy (PPG) [22]. Although the attenuation of postoperative symptoms observed in these studies was reproduced in our present study, we failed to detect significant differences in the VAS scores or in any of the DAUGS items between the patient groups with high and low mean ratios of acyl-/total ghrelin concentration. The attenuation of gastrointestinal symptoms and the improvement in appetite after rikkunshito administration may therefore be attributable to other mechanisms associated with the constituents of rikkunshito rather than the one involving ghrelin. Rikkunshito is composed of over 100 compounds, including 8 major constituents, and a prokinetic action via NG-nitro-L-arginine could be considered as another candidate mechanism for the symptom relief [39, 40]. Prokinetic drugs, including erythromycin, cisapride, metoclopramide, and domperidone, may accelerate gastric emptying after gastrectomy and alleviate post-gastrectomy symptoms [40-43]. In adult patients with diabetic gastroparesis, normalization of electrical activity has been seen after domperidone administration [44]. Cisapride has been demonstrated to improve gastric emptying and myoelectrical activity in children with dyspepsia [45]. However, further studies will be needed to reinforce the hypothesis that herbal medicines, such as rikkunshito, act as prokinetic drugs, as there have been few reports objectively evaluating such effects of these agents [17, 46].

Recently, some reports have demonstrated that rikkunshito induces endogenous ghrelin secretion via its flavonoids, which antagonize 5-HT2B and 5-HT2c receptors $[16,33]$. Furthermore, these reports demonstrated that the oral administration of rikkunshito ameliorated anorexia and gastrointestinal dysmotility in rats treated with either cisplatin or selective serotonin reuptake inhibitors, suggesting that 5-HT2 receptor antagonism could be at least partially responsible for the effect of rikkunshito in improving appetite loss $[16,33]$. Rikkunshito attenuated cisplatin-induced gastrointestinal dysfunction through the improvement of ghrelin release that occurred via the 5-HT2B/2C antagonistic action of flavonoids contained in rikkunshito [16]. In general, herbal medicines such as rikkunshito have not been first-choice candidates for treating various diseases, because they consist of multiple components whose pharmacological function and active ingredients have not been elucidated in detail. Therefore, additional mechanistic studies are needed to conclusively determine the effects of rikkunshito in the near future.

Finally, we note that the differences in the VAS scores, the total DAUGS scores, and scores for various DAUGS items before and after the administration of rikkunshito were significantly different, but perhaps the differences were not spectacular. It is not possible at this stage to deny the possibility that the improvements observed had merely been placebo effects. On the other hand, the statistically significant improvements observed after only 4 weeks of drug intake could indicate the favorable potential of this drug. A prospective placebo-controlled randomized trial testing longer exposure to rikkunshito and including other useful parameters such as body weight, endoscopic findings, and hematological tests as endpoints is needed to further pursue our understanding of this drug.

In conclusion, our study showed, for the first time, that the administration of rikkunshito resulted in the attenuation of post-gastrectomy symptoms, possibly through an increase in the ratio of the acyl-/total ghrelin concentration. Rikkunshito is potentially useful to minimize gastrointestinal symptoms after gastrectomy. A well-designed multicenter randomized trial is warranted to confirm the present findings.

Conflict of interest The authors declare no conflict of interest.

\section{References}

1. Kakizoe T, Yamaguchi N, Mitsuhashi F, et al. Cancer statistics in Japan 2001. Tokyo: Foundation for Promotion of Cancer Research; 2001.

2. Okabayashi T, Gotoda T, Kondo H, et al. Early carcinoma of the gastric cardia in Japan: is it different from that in the West? Cancer. 2000;89:2555-9.

3. Hioki K, Nakane Y, Yamamoto Y, et al. Surgical strategy for early gastric cancer. Br J Surg. 1990;77:1330-40.

4. Sawai K, Takahashi T, Suzuki H. New trends in surgery for gastric cancer in Japan. J Surg Oncol. 1994;56:221-6.

5. Calman KC. Quality of life in cancer patients: a hypothesis. J Med Ethics. 1984;10:124-7.

6. Blazeby JM. The role of quality of life assessment in gastric cancer. Jpn J Clin Oncol. 2000;30:246.

7. Kaptein AA, Morita S, Sakamoto J. Quality of life in gastric cancer. World J Gastroenterol. 2005;11:3189-96.

8. Kojima M, Hosoda H, Date Y, et al. Ghrelin is a growthhormone-releasing acylated peptide from stomach. Nature. 1999; 402:656-60. 
9. Nakazato M, Murakami N, Date Y, et al. A role for ghrelin in the central regulation of feeding. Nature. 2001;409:194-8.

10. Van der Lely AJ, Tschop M, Heiman ML, et al. Biological physiological, pathophysiological, and pharmacological aspects of ghrelin. Endocr Rev. 2004;25:426-57.

11. Ariyasu H, Iwakura H, Yamada G, et al. Efficacy of ghrelin as a therapeutic approach for age related physiological changes. Endocrinology. 2008;149:3722-8.

12. Akamizu T, Kangawa K. Translational research on the clinical applications of ghrelin. Endocr J. 2006;53:585-91.

13. Adachi S, Takiguchi S, Okada K, et al. Effects of ghrelin administration after total gastrectomy: a prospective, randomized, placebocontrolled phase II study. Gastroenterology. 2010;138:1312-20.

14. Yamamoto K, Takiguchi S, Miyata H, et al. Randomized phase II study of clinical effects of ghrelin after esophagectomy with gastric tube reconstruction. Surgery. 2010;141:31-8.

15. Matsumura T, Arai M, Yonemitsu Y, et al. The traditional Japanese medicine rikkunshito increases the plasma level of ghrelin in humans and mice. J Gastroenterol. 2010;45:300-7.

16. Takeda H, Sadakane C, Hattori T, et al. Rikkunshito, an herbal medicine, suppresses cisplatin-induced anorexia in rats via 5-HT2 receptor antagonism. Gastroenterology. 2008;134:2004-13.

17. Tatsuta A, Ishii H. Effect of treatment with Lin-Jun-Zi-Tang (TJ-43) on gastric emptying and gastrointestinal symptoms in dyspeptic patients. Aliment Pharmacol Ther. 1993;7:459-62.

18. Oka T, Tamagawa Y, Tamagawa Y, et al. Rikkunshito attenuates adverse gastrointestinal symptoms induced by fluvoxamine. Biopsychosoc Med. 2007;15:1-21.

19. Kawahara H, Kubota A, Hasegawa T, et al. Effect of rikkunshito on the clinical symptoms and esophageal acid exposure in children with symptomatic gastroesophageal reflux. Pediatr Surg Int. 2007;23:1001-5.

20. Kawahara H, Mitani Y, Nomura M, et al. Impact of rikkunshito, an herbal medicine, on delayed gastric emptying in profoundly handicapped patients. Pediatr Surg Int. 2009;25:987-90.

21. Yagi M, Homma S, Kubota M, et al. The herbal medicine rikkunshito stimulates and coordinates the gastric myoelectric activity in post-operative dyspeptic children after gastrointestinal surgery. Pediatr Surg Int. 2004;19:760-5.

22. Takahashi T, Endo S, Nakajima K, Souma Y, Nishida T. Effect of rikkunshito, a Chinese herbal medicine, on stasis in patients after pylorus-preserving gastrectomy. World J Surg. 2009;33:296-302.

23. Tomono H, Ito $\mathrm{Y}$, Watanabe T. Successful antiemetic treatment of TSUMURA rikkunshito extract granules for ethical use in additional to other antiemetic agents in neoadjuvant chemotherapy for an advanced breast cancer patient. Gan To Kagaku Ryoho. 2006;33:1129-31.

24. Kido T, Nakai Y, Kase Y, et al. Effects of rikkunshito, a traditional Japanese medicine, on the delay of gastric emptying induced by $\mathrm{N}(\mathrm{G})$-nitro-L-arginine. J Pharmacol Sci. 2005;98: 161-7.

25. Takiguchi S, Yamamoto $\mathrm{K}$, Hirao $\mathrm{M}$, et al. A comparison of postoperative quality of life and dysfunction after Billroth I and Roux-en-Y reconstruction following distal gastrectomy for gastric cancer: results from a multi-institutional RCT. Gastric Cancer. 2012;15(2):198-205.

26. Tominaga $\mathrm{K}$, Kido $\mathrm{T}$, Ochi $\mathrm{M}$, et al. The traditional Japanese medicine rikkunshito promotes gastric emptying via the antagonistic action of the 5-HT3 receptor pathway in rats. Evid Based Complement Altern Med. 2009. doi:10.1093/ecam/nep173

27. Nakamura M, Hosoya Y, Yano M, et al. Extent of gastric resection impacts patient quality of life: the Dysfunction after Upper Gastrointestinal Surgery for Cancer (DAUGS32) scoring system. Ann Surg Oncol. 2011;18:314-20.

28. Aaronson NK, Ahmedzai S, Bergman B, et al. The European Organization for Research and Treatment of Cancer QLQ-C30: a quality-of-life instrument for use in international clinical trials in oncology. J Natl Cancer Inst. 1993;85:365-76.

29. Akamizu T, Shinomiya T, Irako T, et al. Separate measurement of plasma levels of acylated and desacyl ghrelin in healthy subjects using a new direct ELISA assay. J Clin Endocrinol Metab. 2005;90:6-9.

30. Kusunoki H, Haruma K, Hata J, et al. Efficacy of rikkunshito, a traditional Japanese medicine (Kampo), in treating functional dyspepsia. Intern Med. 2010;49:2195-202.

31. Goso Y, Ogata Y, Ishihara K, et al. Effects of traditional herbal medicine on gastric mucin against ethanol-induced gastric injury in rats. Comp Biochem Physiol C Pharmacol Toxicol Endocrinol. 1996;113:17-21.

32. Miwa H, Koseki J, Oshima T, et al. Rikkunshito, a traditional Japanese medicine, may relieve abdominal symptoms in rats with experimental esophagitis by improving the barrier function of epithelial cells in esophageal mucosa. J Gastroenterol. 2010;45:478-87.

33. Fujitsuka N, Asakawa A, Hayashi M, et al. Selective serotonin reuptake inhibitors modify physiological gastrointestinal motor activities via 5-HT2c receptor and acyl ghrelin. Biol Psychiatry. 2009;65:748-59.

34. Takachi K, Doki Y, Ishikawa O, et al. Postoperative ghrelin levels and delayed recovery from body weight loss after distal or total gastrectomy. J Surg Res. 2006;130:1-7.

35. Hosoda H, Kojima K, Mizushima T, et al. Structural divergence of human ghrelin. Identification of multiple ghrelin-derived molecules produced by post-translational processing. J Biol Chem. 2003;278:64-70.

36. Date Y, Kojima M, Hosoda H, et al. Ghrelin, a novel growth hormone-releasing acylated peptide, is synthesized in a distinct endocrine cell type in the gastrointestinal tract of rats and humans. Endocrinology. 2000;141:4255-61.

37. Hiejima H, Nishi $Y$, Hosoda $H$, et al. Regional distribution and the dynamics of $n$-decanoyl ghrelin, another acyl-form of ghrelin, upon fasting in rodents. Regul Pept. 2009;156:47-56.

38. Sadakane C, Muto S, Nakagawa K, et al. 10-Gingerol, a component of rikkunshito, improves cisplatin-induced anorexia by inhibiting acylated ghrelin degradation. Biochem Biophys Res Commun. 2011;2(412):506-11.

39. Hashimoto K, Kase Y, Murata P, et al. Pharmacological evaluation of Shokyo and Kankyo (1). Biol Pharm Bull. 2002;25: 1183-7.

40. Nakabayashi T, Mochiki E, Kamiyama Y, et al. Erythromycin induces pyloric relaxation accompanied by a contraction of the gastric body after pylorus-preserving gastrectomy. Surgery. 2003; 133:647-55.

41. Abell TL, Camilleri M, DiMagno EP, et al. Long-term efficacy of oral cisapride in symptomatic upper gut dysmotility. Dig Dis Sci. 1991;36:616-20.

42. Soykan I, Sarosiek I, McCallum RW. The effect of chronic oral domperidone therapy on gastrointestinal symptoms, gastric emptying, and quality of life in patients with gastroparesis. Am J Gastroenterol. 1997;92:976-80.

43. Loo FD, Palmer DW, Soergel KH, et al. Gastric emptying in patients with diabetes mellitus. Gastroenterology. 1984;86: 485-94.

44. Koch KL, Stern RM, Stewart WR, et al. Gastric emptying and gastric myoelectrical activity in patients with diabetic gastroparesis: effect of long-term domperidone treatment. Am J Gastroenterol. 1989;84:1069-75.

45. Riezzo G, Cucchiara S, Chiloiro M, et al. Gastric emptying and myoelectrical activity in children with non-ulcer dyspepsia. Effect of cisapride. Dig Dis Sci. 1995;40:1428-34.

46. Hayakawa T, Arakawa T, Kase Y, et al. Liu-Jun-Zi-Tang, a kampo medicine, promotes adaptive relaxation in isolated guinea pig stomachs. Drugs Exp Clin Res. 1999;25:211-8. 